\title{
High-quality-factor Bragg onion resonators with omnidirectional reflector cladding
}

\author{
Yong Xu, Wei Liang, and Amnon Yariv \\ Department of Applied Physics, MS 128-95, California Institute of Technology, Pasadena, California 91125
}

\author{
J. G. Fleming and Shawn-Yu Lin
}

Sandia National Laboratories, Albuquerque, New Mexico 87185-1080

Received July 1, 2003

\begin{abstract}
We propose to approximate a spherically symmetric Bragg resonator in an onionlike geometry. We develop a transfer-matrix theory for calculation of the quality factors and analyze the effect of the onion stem on cavity $Q$ factors. We find that it is possible to achieve significant inhibition of spontaneous emission in an onion resonator with omnidirectional cladding layers. (C) 2003 Optical Society of America

OCIS codes: $230.5750,220.4000$.
\end{abstract}

With recent advancements in microfabrication techniques, there has been considerable interest in creating an optical cavity with a small modal volume and a large $Q$ factor, in which it should be possible to funnel a significant fraction of the spontaneous emission into a desired cavity mode, ${ }^{1}$ which is critical for realizing single-photon devices. ${ }^{2}$ A silica microsphere can support whispering-gallery modes with quality factors as high as $10^{9} .{ }^{3}$ However, the diameter of such a microsphere typically is greater than $10 \mu \mathrm{m}$, since smaller diameters result in a rapidly decreasing cavity $Q$ factor. ${ }^{3}$ In the case of semiconductor micropillars the modal volume can become very small [of the order of $\left.(\lambda / n)^{3}\right]$, yet fabrication imperfections limit the $Q$ factors to a much lower value of a few thousands. ${ }^{2}$ Recently, a few theoretical discussions of Bragg resonators with perfect spherical symmetry have been presented.,5 Such resonators, however, are difficult to realize. In this Letter we propose to approximate such spherically symmetric Bragg resonators with an onionlike geometry (as shown in Fig. 1), using a process developed for the fabrication of silicon-based miniature Bragg fibers. ${ }^{6}$

The fabrication process of the Bragg onion resonator begins with the introduction of a cylindrical opening in a silicon wafer, which also defines the onion stem in Fig. 1. Subsequently, isotropic etching is used to create a hollow spherical cavity at the end of the stem section. Finally, with chemical vapor deposition the Bragg mirror stacks shown in Fig. 1 can be constructed by the deposition of $\mathrm{Si}$ from silane, $\mathrm{Si}_{3} \mathrm{~N}_{4}$ from dichlorosilane and ammonia, and $\mathrm{SiO}_{2}$ from the thermal oxidation of silicon. A particularly appealing advantage of the onion design is that we can introduce a wide range of light emitters, such as gas-phase neutral atoms and organic molecules, into the onion core through the stem section. Additionally, by choosing a Bragg cladding composed of $\mathrm{Si} / \mathrm{SiO}_{2}$ pairs, which have a large index contrast $\left(n_{\mathrm{Si}}=3.5, n_{\mathrm{SiO}_{2}}=1.5\right)$, we can construct an omnidirectional reflector cladding, ${ }^{7}$ which is important for inhibiting spontaneous emission, as we shall see later in this Letter.
We first present a transfer-matrix formalism for the calculation of resonant frequencies and quality factors of optical modes in a Bragg resonator with complete spherical symmetry. Our method does not use a coupled-mode approximation (as in Ref. 4) and does not rely on a cumbersome calculation of sphericalreflection coefficients (as in Ref. 5). In our approach we assume a time dependence of $\exp (-i \omega t)$ and allow the frequency $\omega$ to be a complex number, the imaginary part of which accounts for the modal loss. In spherical coordinates we can express the general solution to the Maxwell equations in the $n$th concentric dielectric layer as a superposition of various multipole components, each characterized by the angular quantum numbers $l$ and $m$. For example, the electric field can be written $a^{8}$

$$
\mathbf{E}=Z_{n} \sum_{l} \sum_{m=-l}^{l}\left\{\frac{i}{k_{n}} \nabla \times\left[f_{l}\left(k_{n} r\right) \mathbf{X}_{l m}\right]+g_{l}\left(k_{n} r\right) \mathbf{X}_{l m}\right\}
$$

where, with $\epsilon_{n}$ as the relative dielectric constant of the $n$th layer, $Z_{n}=\left(\mu_{0} / \epsilon_{0} \epsilon_{n}\right)^{1 / 2}$ is the material impedance and $k_{n}=\sqrt{\epsilon_{n}} \omega / c$. For structures with complete spherical symmetry the components in Eq. (1) with different $l$ and $m$ decouple, and we can classify various optical modes as $\mathrm{TE}_{l m}$ or $\mathrm{TM}_{l m}$ according to their angular quantum numbers $l$ and $m$ and the polarization state, with the TM mode referring to the first term on the right-hand side of Eq. (1) and the TE mode given by

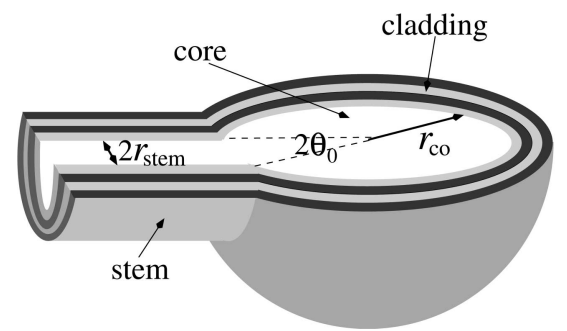

Fig. 1. Schematics of a Bragg onion resonator. 
the second term. ${ }^{4}$ The function $\mathbf{X}_{l m}$, which describes the angular dependence of a $\mathrm{TE}_{l m}$ or $\mathrm{TM}_{l m}$ mode, is given by $\mathbf{X}_{l m}=\left[\mathbf{r} \times \nabla Y_{l m}(\theta, \phi)\right] /\left\{i[l(l+1)]^{1 / 2}\right\}$, where $Y_{l m}(\theta, \phi)$ is a spherical harmonic function. ${ }^{8}$ In the $n$th layer the radial modal dependence is given by a linear combination of the spherical Hankel function of the first kind $\left[h_{l}^{(1)}\left(k_{n} r\right)\right]$ and the second kind $\left[h_{l}^{(2)}\left(k_{n} r\right)\right]$; i.e., $f_{l}\left(k_{n} r\right)=A_{n} h_{l}^{(1)}\left(k_{n} r\right)+B_{n} h_{l}^{(2)}\left(k_{n} r\right)$, and $\left.g_{l}\left(k_{n} r\right)=C_{n} h_{l}^{(1)}\left(k_{n} r\right)+D_{n} h_{l}^{(2)}\left(k_{n} r\right)\right)^{5,8}$ The magnetic field can be expressed similarly. With these considerations and applying the continuity condition to the transverse electromagnetic field across the interface between the $n$th and the $(n+1)$ th layer, we find the following matrix relation for a $\mathrm{TM}_{l m}$ mode:

$$
M_{l}\left(Z_{n}, k_{n}, r_{n}\right)\left(\begin{array}{l}
A_{n} \\
B_{n}
\end{array}\right)=M_{l}\left(Z_{n+1}, k_{n+1}, r_{n}\right)\left(\begin{array}{c}
A_{n+1} \\
B_{n+1}
\end{array}\right),
$$

$$
\begin{aligned}
& M\left(Z_{n}, k_{n}, r_{n}\right) \\
& =\left[\begin{array}{cc}
u_{n} h_{l}^{(1)}\left(k_{n} r_{n}\right) & u_{n} h_{l}^{(2)}\left(k_{n} r_{n}\right) \\
\left.v_{n} \frac{\partial\left[r h_{l}^{(1)}\left(k_{n} r\right)\right]}{\partial r}\right|_{r_{n}} & \left.v_{n} \frac{\partial\left[r h_{l}^{(2)}\left(k_{n} r\right)\right]}{\partial r}\right|_{r_{n}}
\end{array}\right],
\end{aligned}
$$

where the parameters $u_{n}$ and $v_{n}$ are defined as $u_{n}=1$ and $v_{n}=Z_{n} / k_{n}$. For a $\mathrm{TE}_{l m}$ mode Eqs. (2) and (3) still hold if we substitute the vectors $\left[A_{n}, B_{n}\right]$ and $\left[A_{n+1}, B_{n+1}\right]$ with $\left[C_{n}, D_{n}\right]$ and $\left[C_{n+1}, D_{n+1}\right]$, respectively, and use $u_{n}=Z_{n}$ and $v_{n}=1 / k_{n}$.

For a Bragg onion resonator with a finite number of cladding layers we can apply Eqs. (2) and (3) iteratively to relate the coefficients within the onion core $\left(A_{\mathrm{co}}, B_{\mathrm{co}}\right.$, $C_{\text {co }}$, and $\left.D_{\text {co }}\right)$ to those outside the onion ( $A_{\text {out }}, B_{\text {out }}, C_{\text {out }}$, and $\left.D_{\text {out }}\right)$. For a TM mode this leads to

$$
\left(\begin{array}{l}
A_{\text {out }} \\
B_{\text {out }}
\end{array}\right)=\left[\begin{array}{ll}
T_{11} & T_{12} \\
T_{21} & T_{22}
\end{array}\right]\left(\begin{array}{l}
A_{\text {co }} \\
B_{\text {co }}
\end{array}\right)
$$

A similar matrix relation can be derived for a TE mode. The onion cavity modes should satisfy the following boundary conditions: The core electromagnetic field must be finite, and therefore $A_{\text {co }}=B_{\text {co }}$ and $C_{\mathrm{co}}=D_{\mathrm{co}}$, and only the outgoing wave exists outside the onion, which yields $B_{\text {out }}=0$ and $D_{\text {out }}=0$. Substituting these two conditions into Eq. (4), we see that finding the resonant mode of the onion cavity is mathematically equivalent to finding the complex $\omega$ that satisfies $T_{21}+T_{22}=0$. The real part of the solution yields the modal resonance frequency, whereas the imaginary part yields the cavity $Q$ factor through $Q=\operatorname{Re}(\omega) /[2 \operatorname{Im}(\omega)]$.

We now apply our transfer-matrix formalism to investigate the modal spectrum of a specific onion structure. Our example has an air core (with a radius of $r_{\mathrm{co}}=3.47 \mu \mathrm{m}$ ) surrounded by eight Bragg cladding pairs. Each cladding pair is composed of a silicon layer with a refractive index of $n_{\mathrm{Si}}=3.5$ and a thickness of $L_{\mathrm{Si}}=0.111 \mu \mathrm{m}$, followed by a layer of $\mathrm{SiO}_{2}$ with a refractive index of $n_{\mathrm{SiO}_{2}}=1.5$ and a thickness of $L_{\mathrm{SiO}_{2}}=0.258 \mu \mathrm{m}$. In this design the cladding layers form a quarter-wave stack at $\lambda \approx 1.55 \mu \mathrm{m}$. In Figs. 2(a) and 2(b) we show the quality factor of the onion modes with $l \leq 7$ in the wavelength range of $1.35 \mu \mathrm{m} \leq \lambda \leq 1.70 \mu \mathrm{m}$. Since modes with the same $l$ but different $m$ are degenerate, ${ }^{4,5}$ we generally choose $m=0$ for the calculations in Fig. 2. Notice that with only eight pairs of Bragg cladding layers we can achieve cavity $Q$ values close to $8 \times 10^{6}$. In Fig. 2(c) we plot the $Q$ factors of the $\mathrm{TM}_{20}$ mode as a function of Bragg cladding pair number $N_{\text {clad }}$ and find an excellent exponential dependence of $Q \propto D^{N_{\text {clad }}}$. Fitting the numerical results in Fig. 2(c), we find a constant of $D_{\mathrm{TM}_{20}}=5.41$ for the $\mathrm{TM}_{20}$ mode. We observe that this factor is close to $\left(n_{\mathrm{Si}} / n_{\mathrm{SiO}_{2}}\right)^{2}$, which equals 5.44. This similarity can be attributed to the fact that the leakage through the quarter-wave stack of the onion cladding is reduced by a factor of $\left(n_{\mathrm{Si}} / n_{\mathrm{SiO}_{2}}\right)^{2}$ with each additional $\mathrm{Si} / \mathrm{SiO}_{2}$ pair, which improves the quality factor by the same amount.

The previous analysis applies to Bragg resonators with perfect spherical symmetry. The presence of an onion stem, which breaks the spherical symmetry, leads to an additional radiation loss, which we characterize by the quantity $Q_{\text {stem }}$, defined as $\omega E_{\text {cavity }} / P_{\text {stem }}$, where $E_{\text {cavity }}$ is the electromagnetic energy stored in the onion core and $P_{\text {stem }}$ is the power leakage through the onion stem. If $Q_{\text {stem }}$ is much larger than the $Q$ factor of the onion without the stem, we can ignore the presence of the stem in Fig. 1 and treat it as a perfectly spherical onion. If the opposite is true, then the cavity loss is dominated by the photon leakage through the onion stem. From the previous analysis we can picture the optical fields within the onion core as composed of an incoming and an outgoing
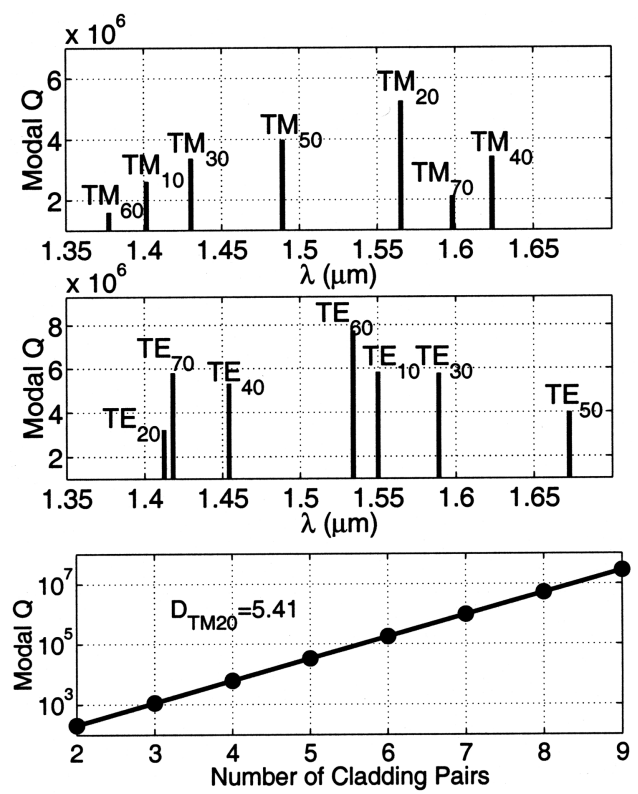

Fig. 2. (a), (b) Wavelength and $Q$ factor of the onion resonator modes. (c) Quality factor of the $\mathrm{TM}_{20}$ modes as a function of the Bragg cladding pair number. The filled circles represent the analytical results, whereas the solid line represents the numerical fit of $Q \propto D^{N_{\text {clad }}}$. 


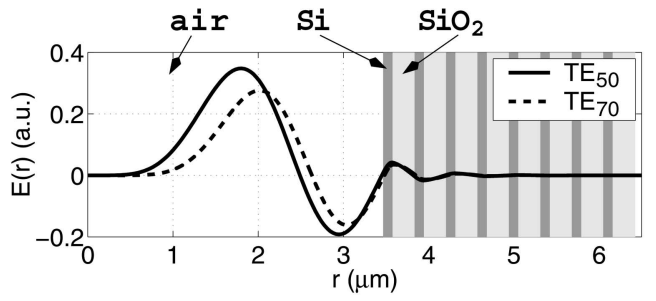

Fig. 3. Radial dependence of the $\mathrm{TE}_{50}$ and $\mathrm{TE}_{70}$ field.

spherical wave with equal amplitude but in opposite directions, with an angular radiation pattern given by $\left|\mathbf{X}_{l m}(\theta, \phi)\right|^{2}{ }^{8} \quad$ For onions with a core size comparable with the photon wavelength we can estimate $Q_{\text {stem }}$ as $1 /\left[\left|\mathbf{X}_{l m}\left(\theta_{0}, \phi\right)\right|^{2} \Delta \Omega_{\text {stem }}\right]$, where $\theta_{0}$ is defined in Fig. 1 and $\Delta \Omega_{\text {stem }}$ is the solid angle spanned by the stem. From this expression we find that among the dipole modes $(l=1)$ the one with $m=0$ has the lowest stem loss, with $Q_{\text {stem }}=4 /\left(3 \theta_{0}^{4}\right)$ (assuming $\theta_{0} \ll \pi / 2$ ). However, for $l \geq 2$ those with $m= \pm l$ have the highest $Q_{\text {stem }}$, which is given by

$$
Q_{\text {stem }}=\frac{(l+1)\left(2^{l} l !\right)^{2}}{(2 l+1) ! l} \frac{1}{\theta_{0}^{2 l}} .
$$

Assuming a stem radius of $0.5 \mu \mathrm{m}$ and a core radius of $3.47 \mu \mathrm{m}$, we find that for the resonant modes with $l>4$ and $m= \pm l$ the $Q_{\text {stem }}$ exceeds $10^{7}$, which means that the presence of an onion stem has little effect on the cavity $Q$ factors. It also implies that for $l>4$ the onion stem does not significantly modify the electromagnetic fields within the onion core from those in a perfectly spherically symmetric Bragg resonator.

In Fig. 3 we show the radial dependence of the $\mathrm{TE}_{50}$ and $\mathrm{TE}_{70}$ modes. For both modes we observe that the electric field is zero at the air-silicon interface, which is similar to the case of a hollow spherical metallic cavity. This is not a coincidence, since the onion cladding forms an omnidirectional reflector and behaves like a perfect metal in the wavelength range of $1.34 \mu \mathrm{m}<\lambda<1.76 \mu \mathrm{m} .{ }^{7}$ Within the omnidirectional bandgap (i.e., $1.34 \mu \mathrm{m}<\lambda<1.76 \mu \mathrm{m}$ ) any light incident on the omnidirectional cladding of the onion resonator is reflected, irrespective of the incidence angle. ${ }^{7}$ As a result, the Bragg cladding completely isolates the onion core from the outside free space. An interesting situation occurs in the off-resonance case, in which the light-emission frequency of the active medium (in the onion core) lies outside all the cavity resonances but still within the omnidirectional bandgap of the cladding layers. Under these conditions, the spontaneous emission of the light emitters in the onion core are greatly suppressed, since the light emitters can couple only to the cladding modes that are confined mainly in the onion cladding or to the free-space modes through the onion stem. Significantly suppressing the spontaneous emission makes it possible to explore the higher-order coupling between the light emitters, which can lead to some interesting quantum optics phenomena. ${ }^{9}$ In this respect the onion cavity resembles more a defect cavity in a three-dimensional photonic crystal, which can also significantly inhibit spontaneous emission. However, the onion structures have considerable advantages in their design simplicity and practical fabrication process.

The authors are thankful for support from the Office of Naval Research (Y. S. Park) and the Defense Advanced Research Projects Agency (D. Honey and R. Athale). Y. Xu's e-mail address is yong@ its.caltech.edu.

\section{References}

1. E. M. Purcell, Phys. Rev. 69, 681 (1946).

2. J. M. Gérard, B. Sermage, B. Gayral, B. Legrand, E. Costard, and V. Thierry-Mieg, Phys. Rev. Lett. 81, 1110 (1998).

3. J. R. Buck and H. J. Kimble, Phys. Rev. A 67, 033806 (2003).

4. D. Brady, G. Papen, and J. E. Sipe, J. Opt. Soc. Am. B 10, 644 (1993).

5. M. A. Kaliteevski, S. Brand, R. A. Abram, and V. V. Nikolaev, J. Mod. Opt. 48, 1503 (2001).

6. J. G. Fleming, S.-Y. Lin, and R. Handley, in Proceedings of the Solid-State Sensor, Actuator, and Microsystems Workshop (Transducer Research Foundation, Cleveland, Ohio, 2002), p. 173.

7. J. N. Winn, Y. Fink, S. Fan, and J. D. Joannopoulos, Opt. Lett. 23, 1573 (1998).

8. J. D. Jackson, Classical Electrodynamics, 3rd ed. (Wiley, New York, 1999).

9. S. John and T. Quang, Phys. Rev. Lett. 74, 3419 (1995). 